Journal of the Optical Society of Korea, Vol. 18, No. 2, pp. 188-193 April 2014

\title{
General Theory of Wave Scattering by Two Separated Particles
}

\author{
Byong Chon Park', Myung-Whun $\mathrm{Kim}^{2}$, and Jin Seung Kim²* \\ ${ }^{I}$ Division of Industrial Metrology, Korea Research Institute of Standards and Science, \\ Daejeon 305-340, Korea \\ ${ }^{2}$ Institute of Photonics and Information Technology, Department of Physics, Chonbuk National University, \\ Jeonju 561-756, Korea
}

※ In our paper, there was a typographic mistake in the grant number cited in the Acknowledgment. The Acknowledgment with correct grant number is below:

\section{ACKNOWLEDGMENT}

This work was supported in part by the IT R\&D program of MKE/KEIT (grant number: 10039226) and Nano-material Technology Development Program through the National Research Foundation (grant number: 2011-0030235), Korea. J. S. Kim is also supported by the International Collaborative Research Funds of Chonbuk National University, 2009.

*Corresponding author: jin@chonbuk.ac.kr 